\title{
Multi-centric study of psychological disturbances among health care workers in tertiary care centers of western India during the COVID-19 pandemic
}

\author{
Ankita Vikrambhai Patel ${ }^{1}$, Dhiraj Dilipkumar Kandre², Prakash Mehta ${ }^{3}$, Aalapi Prajapati \\ Bhumika Patel ${ }^{3}$, Sanjiv Prajapati ${ }^{4}$ \\ ${ }^{1}$ GMERS Medical College and Hospital, Vadnagar, Gujarat, India \\ ${ }^{2}$ GMERS Medical College, Himmatnagar, Gujarat, India \\ ${ }^{3}$ GMERS Medical College, Sola, Ahmedabad, Gujarat, India \\ ${ }^{4}$ Cardiologist in Multi-Speciality Hospital, India
}

Neuropsychiatria i Neuropsychologia 2020; 15, 3-4: 89-100

\author{
Address for correspondence: \\ Dhiraj Dilipkumar Kandre, MD \\ GMERS Medical College \\ Himmatnagar, Gujarat, India \\ phone: 9428871335 \\ e-mail: kandre.d.j@gmail.com
}

\begin{abstract}
Introduction: The world has been facing a pandemic of COVID-19, and while dealing with this pandemic is not easy for anyone, it is especially hard on health care workers (HCWs) as they are the front-line warriors.

Aim of the study: To assess the psychological disturbances that the COVID-19 pandemic has inflicted on healthcare workers in India and to assess the correlation between different parameters including socio-demographic variables, job-related variables, personal history and psychological disturbances such as perceived stress, depression and anxiety.

Material and methods: Health care workers from a few tertiary care institutes (government as well as private sectors, which are caring for patients with COVID-19) in a western state of India were invited to participate with a self-administered online questionnaire. That questionnaire consisted of self-report scales including Fear of COVID-19 Scale, the Depression, Anxiety and Stress Scale 21 items (DASS 21), and the Perceived Stress Scale with socio-demographic, job-related and personal details.

Results: Out of 302 subjects, on the Perceived Stress Scale, 101 (33.44\%) reported low, 185 (61.26\%) moderate and $16(5.30 \%)$ high levels of stress. On DASS 21, depression was reported by $56(18.54 \%)$ subjects, $60(19.87 \%)$ subjects were found to have anxiety and $50(16.56 \%)$ subjects reported to have stress. Perceived stress score on Perceived Stress Scale (PSS) was significantly correlated with the depression, anxiety and stress score on DASS 21. A few risk factors such as female gender, single marital status, past history of psychiatric illness, working as interns and residents doctors with lesser experience and high risk duty areas such as COVID-19 duties in a particular situation were significantly associated with psychological disturbances such as stress, depression and anxiety.

Conclusions: We observed significant psychological impacts of the COVID-19 pandemic on HCWs as well as a few risk factors related to it. Supporting the mental health of HCWs is a critical part of the public health response to maintain an adequate workforce and to maximize the ability of HCWs to face this pandemic.
\end{abstract}

Key words: health care workers, COVID-19, psychological disturbances.

\section{Introduction}

Since December 2019 the world has been facing an outbreak of a novel infectious disease known as coronavirus disease 2019 (COVID-19), that has rapidly spread globally with the number of confirmed cases increasing every day and has now been declared as a pandemic by the World Health Organization (2020). The current outbreak of the novel coronavirus SARS-CoV-2 has its epicenter in Hubei Province of the People's Republic of China (Velavan and Meyer 2020). COVID-19 is caused by a novel, enveloped single-stranded RNA virus, severe acute respiratory syndrome coronavirus-2 (SARS-CoV-2). SARS-CoV-2 is the seventh known coronavirus in humans and belongs to the same phylogenetic family as the 2002 SARS and the 2012 Middle East respiratory syndrome coronavirus (MERSCoV-2). SARS-CoV-2 is presumed - but not 
confirmed - to have originated in bats given a remarkable (89-96\%) genomic homology to bat coronaviruses (Andersen et al. 2020). Recent data show that there are 11 million cases of COVID-19 worldwide with 524000 deaths. In India, there have been 262000 cases of COVID-19 with 18213 deaths due to COVID-19. Gujarat has reported the $5^{\text {th }}$ highest number of cases behind the states of Maharashtra, Tamil Nadu, Delhi and Chennai. In Gujarat 33913 cases of COVID-19 have been reported so far with 1886 deaths (COVID-19 dashboard India on date 3 July 2020).

Dealing with this pandemic is not easy for anyone, but it is especially hard on health care workers (HCWs) as they are the front-line warriors. Some major concerns for HCWs include: worry that they may carry the coronavirus home and infect loved ones; the fear and uncertainty of a heightened risk of infection; a dwindling or inadequate supply of personal protective equipment (PPE); frequently changing recommendations from local leaderships, medical and health experts and political leaders; unusually high and increasing demands to work longer hours as their colleagues become sick or are quarantined; balancing their commitment to help others with an understandable commitment to protect themselves and their loved ones; widespread media coverage, lack of specific drugs and feelings of being inadequately supported. These may all contribute to the mental burden of these health care workers. Previous studies have reported adverse psychological reactions to the 2003 SARS outbreak among health care workers. Studies showed that those HCWs feared contagion and infection of their family, friends and colleagues, felt uncertainty and stigmatization, reported reluctance to work or were contemplating resignation, and reported experiencing high levels of stress, anxiety and depression symptoms, which could have long-term psychological implications. A survey of 1257 nurses and physicians caring for patients with the disease in China found that these providers (41.5\% of respondents) had significantly more depression, anxiety, insomnia and distress than providers who did not care directly for patients (Lai et al. 2020). Another observational study of $180 \mathrm{HCWs}$ providing direct care for patients with COVID-19 found substantial levels of anxiety and stress that adversely influenced sleep quality and self-efficacy (Xiao et al. 2020).

Data derived from previous epidemics, such as SARS in 2003 and the H1N1 influenza in 2009 , illustrate that the community suffered considerable fear and panic, resulting in a significant psychological impact (Chong et al. 2004; Goulia et al. 2010). Experience from the SARS and $\mathrm{H} 1 \mathrm{~N} 1$ epidemics underline that the psychological strain on healthcare professionals is significant. Therefore, healthcare professionals dealing with COVID-19 are under increased psychological pressure and experience high rates of psychiatric morbidity, resembling the situation during the SARS and H1N1 epidemics (Chong et al. 2004; Brooks et al. 2018). A very recent study among healthcare professionals in a tertiary infectious disease hospital for COVID-19 in China revealed a high incidence of anxiety and stress disorders among frontline medical staff, with nurses having a higher incidence of anxiety than doctors. The disruption of routine clinical practice, the sense of loss of control and the subsequent fear of potential destabilization of the health services have provoked 'overflowing' anxiety and depression among healthcare professionals, a feature which is not uncommon in epidemics (Brooks et al. 2018; Aoyagi et al. 2015). However, in light of the increased psychological pressure of frontline healthcare staff, measures for psychological support and interventions to protect their mental health should be adopted promptly, as shown by previous experience (Goulia et al. 2010; Brooks et al. 2018).

Work-related stress is a potential cause of concern in HCWs and is associated with decreased job satisfaction, days of work, anxiety, depression, sleeplessness, medical errors and near misses. Despite the high prevalence of stress in doctors, and a myriad of physical and mental health consequences, doctors are notoriously reluctant to seek help for themselves. Doctors are also "poor" patients due to maladaptive health behaviors (Adshead 2005; Chambers and Belcher 1992; Forsythe et al. 1999).

The COVID-19 pandemic is an unprecedented significant challenge for health care workers; so it is highly important to acknowledge the psychological impact of this mounting threat on healthcare professionals. Our impression is that the increasing knowledge about preventing and dealing with the disease, and the development of more specific procedural and treatment protocols, alongside educational activities, will contribute to improving the morale of healthcare workers dealing with the pandemic. We have assessed the psychological disturbances that the COVID-19 pandemic has inflicted on healthcare professionals in India and have reviewed the literature around the effect of previous epidemics on frontline healthcare staff. 


\section{Material and methods}

Participant recruitment procedures in detail: HCWs including physicians/consultants, resident doctors, interns, and nursing staff from a few tertiary care institutes (government as well as private sectors, which are caring for patients with COVID-19) in a western state of India, were invited to participate with a self-administered online questionnaire. Those who consented to participate were given the following self-report scales: a semi-structural Performa containing basic socio-demographic details, job-related details and personal history; the Depression, Anxiety and Stress Scale 21 items (DASS 21), Cohen's Perceived Stress Scale (PSS) and the Fear of COVID-19 Scale. Different psychological parameters such as stress, anxiety and depression were assessed in all HCWs. The online self-reported questionnaire developed by the investigators contained questions from above-mentioned scales. All of the selected institutes were sent online forms through email or a WhatsApp link. We collected data from 7 May 20209.00 a.m. to 20 May 202012.00 a.m. Comparison between different parameters such as type of work allotted, socio-demographic data, personal history and severity of psychological disturbances was performed.

The Depression, Anxiety and Stress Scale 21 items is a set of three self-report scales designed to measure the emotional states of depression, anxiety and stress (Lovibond and Lovibond 1995). Each of the three DASS-21 scales contains 7 items, divided into subscales with similar content. Each of the questions has a Likert scale of 0 to 3. Scores for depression, anxiety and stress are calculated by summing the scores for the relevant items. Sum scores are computed by adding up the scores on the items per (sub) scale and multiplying them by a factor of 2 . Sum scores for the total DASS-total scale thus range between 0 and 120, and those for each of the subscales may range between 0 and 42. Each of the questions has a Likert scale of 0 to 3 with a range of 0 to 21 for each domain. The options are never (0), little (1), sometimes (2) and always (3). If the score obtained from the questions of the depression subscale ranges from 0 to 4 , the subject will be in the normal range, a score of 5-6 means mild depression, 7-10 suggests moderate depression, 11-13 severe depression, and 14 and over very severe depression. If the score of the anxiety subscale is between 0 and 3 , the subject is in the normal range, 4-5 suggests mild anxiety, 6-7 moderate anxiety, 8-9 means severe anxiety, while 10 and over shows very severe anxiety. Finally, if the score obtained from the questions of the stress subscale ranges between 0 and 7 , the subject has a normal state, 8-9 shows a mild stress, 10-12 suggests moderate stress, 13-16 severe stress, and a score of 17 and over signifies a very severe level of stress.

The Perceived Stress Scale is the most widely used psychological instrument for measuring the perception of stress (Cohen et al. 1983). The Perceived Stress Scale scores are obtained by reversing responses (e.g., $0=4,1=3,2=2$, $3=1$ and $4=0$ ) to the four positively stated items (items 4, 5, 7, 8) and then summing across all scale items. Individual scores on the PSS can range from 0 to 40 with higher scores indicating higher perceived stress. Scores ranging from 0 to 13 would be considered low stress. Scores ranging from 14 to 26 would be considered moderate stress. Scores ranging from 27 to 40 would be considered high perceived stress.

Fear of COVID-19 Scale (Ahorsu et al. 2020): the Fear of COVID-19 Scale is a seven-item uni-dimensional scale with robust psychometric properties. Initial psychometric results indicated that the FCV-19S had good properties from different types of testing (i.e., Classical Test Theory [CTT] and Rasch analysis). Moreover, the overall score of the summed-up items scores can indicate the severity of the fear of COVID-19. Higher overall scores on the FCV-19S indicate more severe fear of COVID-19.

All data were converted into categorical variables. Correlation between different parameters such as socio-demographic variants, job-related variants, personal history and psychological disturbances such as perceived stress, depression and anxiety were observed using $\chi^{2}$. $P$-values were assumed to be significant at $<0.05$. SPSS 20.0 version was used for statistical calculations.

\section{Results}

Three hundred and two subjects were included in the study. On PSS, $101(33.44 \%)$ reported low stress, $185(61.26 \%)$ moderate and $16(5.30 \%)$ reported a high level of stress (Table 1). Stress on PSS was significantly correlated with gender as female subjects showed a significantly higher level of stress than males (Table 2).

On DASS 21, depression was reported by $56(18.54 \%)$ subjects of whom $18(5.96 \%)$ had mild, 22 (7.28\%) had moderate, 8 (2.65\%) had severe and $8(2.65 \%)$ had extremely severe depression. On the DASS 21 scale, 60 (19.87\%) subjects were found to have anxiety of whom 
$11(3.64 \%)$ had mild, $24(7.95 \%)$ moderate, $12(3.97 \%)$ severe and $13(4.30 \%)$ extremely severe anxiety. On DASS 21, 50 subjects reported to have stress, of whom $13(4.30 \%)$ had mild, $5(1.66 \%)$ had moderate, $13(4.30 \%)$ had se-

Table 1 . Severity of perceived stress

\begin{tabular}{lc} 
PSS score and stress level & $\boldsymbol{N}=\mathbf{3 0 2}$ \\
Low perceived stress (0-13) & 101 \\
\hline Moderate perceived stress (14-26) & 185 \\
\hline High perceived stress (27-40) & 16 \\
\hline PSS - Perceived Stress Scale &
\end{tabular}

vere and $4(1.32 \%)$ had extremely severe stress (Table 3). Perceived stress score on PSS was significantly correlated with depression, anxiety and stress score on DASS 21 (Table 4). No significant association observed between stress on PSS and work related factors (Table 5). Depression DASS 21 was significantly correlated with subjects' working post, work experience and history of psychiatric illness (Table 6). Depression was significantly higher in interns and resident doctors and those who had past history of psychiatric illness. Subjects who had longer work experience had lower risk of depression (Tables 6

Table 2. Association between stress, socio-demographical and personal factors

\begin{tabular}{|c|c|c|c|c|c|}
\hline \multirow{2}{*}{$\begin{array}{l}\text { Socio-demographic } \\
\text { and personal factors }\end{array}$} & \multirow{2}{*}{ Total $(N=302)$} & \multicolumn{3}{|c|}{ PSS } & \multirow{2}{*}{$\chi^{2}$ and $p$-value } \\
\hline & & Low & Moderate & High & \\
\hline \multicolumn{5}{|l|}{ Age } & \multirow{6}{*}{$\begin{array}{l}\chi^{2}=15.01 \\
p=0.058\end{array}$} \\
\hline 21-30 years & 176 & 50 & 114 & 12 & \\
\hline $31-40$ years & 90 & 30 & 57 & 3 & \\
\hline 41-50 years & 11 & 7 & 3 & 1 & \\
\hline $51-60$ years & 15 & 8 & 7 & 0 & \\
\hline$>60$ years & 10 & 6 & 4 & 0 & \\
\hline \multicolumn{5}{|l|}{ Gender } & \multirow{3}{*}{$\begin{array}{c}\chi^{2}=7.1 \\
p=0.027\end{array}$} \\
\hline male & 189 & 71 & 112 & 6 & \\
\hline female & 113 & 30 & 73 & 10 & \\
\hline \multicolumn{5}{|l|}{ Religion } & \multirow{5}{*}{$\begin{array}{l}\chi^{2}=3.59 \\
p=0.73\end{array}$} \\
\hline Hindu & 281 & 96 & 171 & 14 & \\
\hline Muslim & 15 & 4 & 9 & 2 & \\
\hline Christian & 5 & 1 & 4 & 0 & \\
\hline others & 1 & 0 & 1 & 0 & \\
\hline \multicolumn{5}{|l|}{ Marital status } & \multirow{5}{*}{$\begin{array}{l}\chi^{2}=5.86 \\
p=0.44\end{array}$} \\
\hline single & 148 & 45 & 91 & 12 & \\
\hline married & 150 & 55 & 91 & 4 & \\
\hline divorced/separated & 3 & 1 & 2 & 0 & \\
\hline widow/widower & 1 & 0 & 1 & 0 & \\
\hline \multicolumn{5}{|l|}{ Family types } & \multirow{3}{*}{$\begin{aligned} \chi^{2} & =3.39 \\
p & =0.18\end{aligned}$} \\
\hline nuclear & 165 & 51 & 102 & 12 & \\
\hline joint & 137 & 50 & 93 & 4 & \\
\hline \multicolumn{5}{|c|}{ History of physical illnesses } & \multirow{7}{*}{$\begin{array}{l}\chi^{2}=6.78 \\
p=0.74\end{array}$} \\
\hline none & 237 & 84 & 158 & 13 & \\
\hline cardiac illness & 13 & 6 & 7 & 0 & \\
\hline respiratory illnesses & 13 & 3 & 2 & 0 & \\
\hline diabetes & 5 & 4 & 7 & 2 & \\
\hline cardiac + diabetes & 1 & 4 & 10 & 1 & \\
\hline others & 15 & 0 & 1 & 0 & \\
\hline \multicolumn{5}{|c|}{ Past history of psychiatric illness } & \multirow{4}{*}{$\begin{aligned} \chi^{2} & =3.35 \\
p & =0.5\end{aligned}$} \\
\hline yes & 13 & 3 & 8 & 2 & \\
\hline no & 277 & 94 & 170 & 13 & \\
\hline maybe & 12 & 4 & 7 & 1 & \\
\hline
\end{tabular}

PSS-Perceived Stress Scale 
and 7). Anxiety score on DASS 21 significantly correlated with marital status, working post and working area of subjects. Anxiety was higher in single than married subjects. Interns and resident doctors had significantly higher anxiety than others. Subjects who had COVID-19 duties had a higher level of anxiety than others (Tables 8 and 9).

On the Fear of COVID-19 Scale, 105 subjects reported that they had fear of coronavirus. 44 subjects also feared losing their life or dying of COVID-19. 14 subjects also reported that they had sleep disturbance because of worrying regarding getting COVID-19 (Table 10).

\section{Discussion}

In our study, out of 302 subjects, 101 (33.44\%) reported low stress, 185 (61.26\%) moderate stress and $16(5.30 \%)$ reported a high level of stress, on PSS. On DASS 21, 50 subjects reported to have stress, of whom $13(4.30 \%)$ had mild, $5(1.66 \%)$ had moderate, $13(4.30 \%)$ had severe and $4(1.32 \%)$ had extremely severe stress. On DASS 21, depression was reported by $56(18.54 \%)$ subjects, of whom $18(5.96 \%)$ had mild, $22(7.28 \%)$ had moderate, $8(2.65 \%)$ had severe and $8(2.65 \%)$ had extremely severe depression. On the DASS 21 scale, 60 (19.87\%) subjects were found to have anxiety, of whom $11(3.64 \%)$ had mild, $24(7.95 \%)$ moderate, $12(3.97 \%)$ severe and $13(4.30 \%)$ extremely severe anxiety. Stress score on PSS was significantly correlated with depression, anxiety and stress score on DASS 21.

Our results are consistent with a few recent studies. A very recent study among healthcare professionals in a tertiary infectious disease hospital for COVID-19 in China revealed a high incidence of anxiety and stress disorders among
Table 3. Presence of depression, stress and anxiety amongst health care workers

\begin{tabular}{llc}
\hline Depression & normal (0-9) & 246 \\
\cline { 2 - 3 } & mild (10-13) & 18 \\
\cline { 2 - 3 } moderate (14-20) & 22 \\
\cline { 2 - 3 } Anxiety & severe (21-27) & 8 \\
\cline { 2 - 3 } & extremely severe (28+) & 8 \\
\cline { 2 - 3 } & mormal (0-7) & 242 \\
\cline { 2 - 3 } & moderate (10-14) & 11 \\
\cline { 2 - 3 } & severe (15-19) & 24 \\
\cline { 2 - 3 } & extremely severe (20+) & 13 \\
\hline Stress & normal (0-14) & 267 \\
\cline { 2 - 3 } & mild (15-18) & 13 \\
\cline { 2 - 3 } & moderate (19-25) & 5 \\
\cline { 2 - 3 } & severe (26-33) & 13 \\
\cline { 2 - 3 } & extremely severe (34+) & \\
\hline
\end{tabular}

frontline medical staff (Huang et al. 2020). Another study conducted in Singapore showed that out of 500 invited health care workers, 470 (94\%) participated in the study; 68 (14.5\%) participants screened positive for anxiety, $42(8.9 \%)$ for depression, 31 (6.6\%) for stress, and $36(7.7 \%)$ for clinical concern of post-traumatic stress disorder (Tan et al. 2020). The study by Zhang et al. (2020) showed that medical health workers $(n=927)$ had a higher prevalence of insomnia ( $38.4 \%$ vs. $30.5 \%, p<0.01$ ), anxiety $(13.0 \%$ vs. $8.5 \%, p<0.01)$, depression $(12.2 \%$ vs. $9.5 \%, p<0.04)$, somatization $(1.6 \%$ vs. $0.4 \%, p<0.01$ ), and obsessive-compulsive symptoms $(5.3 \%$ vs. $2.2 \%, p<0.01)$ when compared with non-medical health workers $(n=1,255)$.

A very recent Indian study showed that out of 152 study participants, $34.9 \%$ were depressed

Table 4. Correlation between depression, anxiety and stress with perceived stress

\begin{tabular}{|c|c|c|c|c|c|}
\hline \multirow{2}{*}{\multicolumn{2}{|c|}{ DASS 21 scoring }} & \multicolumn{3}{|c|}{ PSS level } & \multirow[t]{2}{*}{$\chi^{2}$ and $p$-value } \\
\hline & & Low $(n=101)$ & Moderate $(n=185)$ & High $(n=16)$ & \\
\hline \multicolumn{5}{|c|}{ Depression } & \multirow{3}{*}{$\begin{array}{c}\chi^{2}=56.66 \\
p<0.00001\end{array}$} \\
\hline absent & 246 & 97 & 146 & 8 & \\
\hline present & 56 & 4 & 39 & 8 & \\
\hline \multicolumn{5}{|l|}{ Anxiety } & \multirow{3}{*}{$\begin{array}{c}\chi^{2}=37.64 \\
p<0.00001\end{array}$} \\
\hline absent & 242 & 95 & 142 & 5 & \\
\hline present & 60 & 6 & 43 & 11 & \\
\hline \multicolumn{5}{|l|}{ Stress } & \multirow{3}{*}{$\begin{array}{l}\chi^{2}=37.91 \\
p<0.0001\end{array}$} \\
\hline absent & 267 & 101 & 158 & 3 & \\
\hline present & 35 & 0 & 27 & 13 & \\
\hline
\end{tabular}

DASS 21 -Depression, Anxiety and Stress Scale 21 items, PSS - Perceived Stress Scale 
Table 5. Association between work-related factors and perceived stress

\begin{tabular}{|c|c|c|c|c|c|}
\hline \multirow[t]{2}{*}{ Work-related details } & \multirow{2}{*}{$\begin{array}{c}\text { Total } \\
(N=302)\end{array}$} & \multicolumn{3}{|c|}{ PSS } & \multirow[t]{2}{*}{$\chi^{2}$ and $p$-value } \\
\hline & & Low & Moderate & High & \\
\hline \multicolumn{5}{|l|}{ Working post } & \multirow{6}{*}{$\begin{array}{l}\chi^{2}=12.4 \\
p=0.13\end{array}$} \\
\hline intern doctors & 57 & 16 & 38 & 3 & \\
\hline resident doctors & 87 & 27 & 51 & 9 & \\
\hline consultants & 114 & 41 & 70 & 3 & \\
\hline nursing staff & 9 & 1 & 8 & 0 & \\
\hline others & 35 & 16 & 18 & 1 & \\
\hline \multicolumn{5}{|l|}{ Work experience } & \multirow{5}{*}{$\begin{array}{l}\chi^{2}=10.6 \\
p=0.101\end{array}$} \\
\hline$<1$ year & 82 & 22 & 53 & 7 & \\
\hline $1-3$ years & 57 & 14 & 39 & 4 & \\
\hline$>3-10$ years & 79 & 28 & 49 & 2 & \\
\hline$>10$ years & 84 & 37 & 44 & 3 & \\
\hline \multicolumn{5}{|l|}{ Duty type } & \multirow{3}{*}{$\begin{aligned} \chi^{2} & =1.78 \\
p & =0.4\end{aligned}$} \\
\hline fixed hours & 284 & 94 & 176 & 14 & \\
\hline shift duties & 18 & 7 & 9 & 2 & \\
\hline \multicolumn{5}{|l|}{ Duty hours/day } & \multirow{5}{*}{$\begin{array}{c}\chi^{2}=10.42 \\
p=0.40\end{array}$} \\
\hline$\leq 5$ hours & 31 & 12 & 18 & 1 & \\
\hline $6-8$ hours & 197 & 65 & 121 & 11 & \\
\hline$>8-12$ hours & 61 & 28 & 30 & 3 & \\
\hline$>12-24$ hours & 13 & 3 & 10 & 0 & \\
\hline \multicolumn{5}{|l|}{ Duty hours/week } & \multirow{3}{*}{$\begin{array}{l}\chi^{2}=0.86 \\
p=0.65\end{array}$} \\
\hline$\leq 48$ hours & 187 & 65 & 111 & 11 & \\
\hline$>48$ hours & 115 & 36 & 74 & 5 & \\
\hline \multicolumn{5}{|l|}{ Working area } & \multirow{7}{*}{$\begin{array}{l}\chi^{2}=3.94 \\
p=0.94\end{array}$} \\
\hline COVID-19 duties & 73 & 21 & 47 & 5 & \\
\hline $\begin{array}{l}\text { non-COVID-19 routi- } \\
\text { ne consultations }\end{array}$ & 130 & 43 & 80 & 7 & \\
\hline $\begin{array}{l}\text { COVID-19 duties + } \\
\text { non-COVID-19 routi- } \\
\text { ne consultations }\end{array}$ & 21 & 8 & 13 & 0 & \\
\hline $\begin{array}{l}\text { emergency duties } \\
\text { (ICU, CCU, PICU, } \\
\text { NICU, etc.) }\end{array}$ & 62 & 22 & 36 & 4 & \\
\hline $\begin{array}{l}\text { lab/paramedics/field } \\
\text { duties }\end{array}$ & 9 & 4 & 5 & 0 & \\
\hline administration & 7 & 3 & 4 & 0 & \\
\hline
\end{tabular}

and $39.5 \%$ and $32.9 \%$ had anxiety and stress, respectively (Chatterjee et al. 2020). Another Indian study by Deblina et al. (2020) showed that there are increased worries and apprehensions among the public regarding acquiring the COVID-19 infection. In that study, approximately, half of the population were healthcare professionals.

In our study, we did not find any correlation between stress and other socio-demographic variants such as age, family types and religion. But stress on PSS was significantly correlated with gender as female subjects showed a significantly higher level of stress than males. Also we observed that depression and anxiety were significantly higher in interns and resident doctors and the depression score was significantly higher in those who had a past history of psychiatric illness. Subjects who had longer work experience had lower risk of depression. Anxiety was higher in single than married subjects. Interns and resident doctors had significantly higher anxiety than 
Table 6. Association between depression, socio-demographic and personal factors

\begin{tabular}{|c|c|c|c|c|}
\hline \multirow{2}{*}{$\begin{array}{l}\text { Socio-demographic and } \\
\text { personal factors }\end{array}$} & \multirow[t]{2}{*}{ Total $(N=302)$} & \multicolumn{2}{|c|}{ DASS Depression } & \multirow[t]{2}{*}{$\chi^{2}$ and $p$-value } \\
\hline & & Absent & Present & \\
\hline \multicolumn{4}{|l|}{ Age } & \multirow{6}{*}{$\begin{array}{l}\chi^{2}=5.25 \\
p=0.26\end{array}$} \\
\hline $21-30$ years & 176 & 137 & 39 & \\
\hline 31-40 years & 90 & 76 & 14 & \\
\hline $41-50$ years & 11 & 10 & 1 & \\
\hline $51-60$ years & 15 & 13 & 2 & \\
\hline$>60$ years & 10 & 10 & 0 & \\
\hline \multicolumn{4}{|l|}{ Gender } & \multirow{3}{*}{$\begin{array}{l}\chi^{2}=1.18 \\
p=0.28\end{array}$} \\
\hline male & 189 & 158 & 31 & \\
\hline female & 113 & 88 & 25 & \\
\hline \multicolumn{4}{|l|}{ Religion } & \multirow{5}{*}{$\begin{array}{l}\chi^{2}=6.8 \\
p=0.07\end{array}$} \\
\hline Hindu & 281 & 232 & 49 & \\
\hline Muslim & 15 & 11 & 4 & \\
\hline Christian & 5 & 2 & 3 & \\
\hline others & 1 & 1 & 0 & \\
\hline \multicolumn{4}{|l|}{ Marital status } & \multirow{5}{*}{$\begin{array}{l}\chi^{2}=7.37 \\
p=0.06\end{array}$} \\
\hline single & 148 & 112 & 36 & \\
\hline married & 150 & 131 & 19 & \\
\hline divorced/separated & 3 & 2 & 1 & \\
\hline widow/widower & 1 & 1 & 0 & \\
\hline \multicolumn{4}{|l|}{ Family types } & \multirow{3}{*}{$\begin{array}{l}\chi^{2}=2.12 \\
p=0.14\end{array}$} \\
\hline nuclear & 165 & 129 & 36 & \\
\hline joint & 137 & 117 & 20 & \\
\hline \multicolumn{4}{|l|}{ History of physical illnesses } & \multirow{7}{*}{$\begin{array}{l}\chi^{2}=3.02 \\
p=0.69\end{array}$} \\
\hline none & 237 & 207 & 48 & \\
\hline cardiac illness & 13 & 9 & 4 & \\
\hline respiratory illnesses & 13 & 5 & 0 & \\
\hline diabetes & 5 & 11 & 2 & \\
\hline cardiac + diabetes & 1 & 13 & 2 & \\
\hline others & 15 & 1 & 0 & \\
\hline \multicolumn{4}{|c|}{ Past history of psychiatric illness } & \multirow{4}{*}{$\begin{array}{l}\chi^{2}=8.35 \\
p=0.015\end{array}$} \\
\hline yes & 13 & 8 & 5 & \\
\hline$\overline{\text { no }}$ & 277 & 231 & 46 & \\
\hline maybe & 12 & 7 & 5 & \\
\hline
\end{tabular}

DASS - Depression, Anxiety and Stress Scale

others. Subjects who had COVID-19 duties had a higher level of anxiety than others who were posted for non-COVID-19 duties.

Our results were consistent with some other studies such as the study by Zhang et al. (2020), which showed that being female, and being at risk of contact with COVID-19 patients were the most common risk factors for insomnia, anxiety, obsessive-compulsive symptoms, and depression. Another study (Brooks et al. 2020) and a study by Kushal et al. (2018) showed that total working years as a health care professional significantly negatively correlated with stress. This may be due to HCWs with more experience having gained various experiences and a strong sense of belonging to the organization; they might experience lesser stress than those with lesser work experience. Also significantly higher anxiety in HCWs with COVID-19 due to many reasons such as the increased workload created by such outbreaks, fears of contagion for themselves and their families, working with new and frequently changing protocols and PPE, caring for patients who are very sick and 
Table 7. Association between work related factors and depression

\begin{tabular}{|c|c|c|c|c|}
\hline \multirow[t]{2}{*}{ Work-related details } & \multirow{2}{*}{$\begin{array}{c}\text { Total } \\
(N=302)\end{array}$} & \multicolumn{2}{|c|}{ DASS Depression } & \multirow{2}{*}{$\begin{array}{c}\chi^{2} \text { and } \\
p \text {-value }\end{array}$} \\
\hline & & Absent & Present & \\
\hline \multicolumn{4}{|l|}{ Working post } & \multirow{6}{*}{$\begin{array}{l}\chi^{2}=10.74 \\
p=0.029\end{array}$} \\
\hline intern doctors & 57 & 42 & 15 & \\
\hline resident doctors & 87 & 67 & 20 & \\
\hline consultants & 114 & 103 & 11 & \\
\hline nursing staff & 9 & 6 & 3 & \\
\hline others & 35 & 28 & 7 & \\
\hline \multicolumn{4}{|l|}{ Work experience } & \multirow{5}{*}{$\begin{array}{l}\chi^{2}=8.62 \\
p=0.034\end{array}$} \\
\hline$<1$ year & 82 & 64 & 18 & \\
\hline $1-3$ years & 57 & 41 & 16 & \\
\hline$>3-10$ years & 79 & 65 & 14 & \\
\hline$>10$ years & 84 & 76 & 8 & \\
\hline \multicolumn{4}{|l|}{ Duty type } & \multirow{3}{*}{$\begin{array}{c}\chi^{2}=0.010 \\
p=0.91\end{array}$} \\
\hline fixed hours & 284 & 232 & 52 & \\
\hline shift duties & 18 & 14 & 4 & \\
\hline \multicolumn{4}{|l|}{ Duty hours/day } & \multirow{5}{*}{$\begin{array}{c}\chi^{2}=2.26 \\
p=0.8\end{array}$} \\
\hline$\leq 5$ hours & 31 & 27 & 4 & \\
\hline 6-8 hours & 197 & 157 & 40 & \\
\hline$>8-12$ hours & 61 & 51 & 8 & \\
\hline$>12-24$ hours & 13 & 11 & 2 & \\
\hline \multicolumn{4}{|l|}{ Duty hours/week } & \multirow{3}{*}{$\begin{aligned} \chi^{2} & =0.003 \\
p & =0.96\end{aligned}$} \\
\hline$\leq 48$ hours & 187 & 152 & 35 & \\
\hline$>48$ hours & 115 & 94 & 21 & \\
\hline \multicolumn{4}{|l|}{ Working area } & \multirow{7}{*}{$\begin{array}{l}\chi^{2}=9.43 \\
p=0.09\end{array}$} \\
\hline COVID-19 duties & 73 & 52 & 21 & \\
\hline non-COVID-19 routine consultations & 130 & 112 & 18 & \\
\hline COVID-19 duties + non-COVID-19 routine consultations & 21 & 15 & 6 & \\
\hline emergency duties (ICU, CCU, PICU, NICU, etc.) & 62 & 53 & 9 & \\
\hline lab/paramedics/field duties & 9 & 8 & 1 & \\
\hline administration & 7 & 6 & 1 & \\
\hline
\end{tabular}

DASS - Depression, Anxiety and Stress Scale, ICU - Intensive Care Unit, CCU - Critical Care Unit, PICU - Pediatric Intensive Care Unit, NICU - Neonatal Intensive Care Unit

quickly deteriorating and caring for colleagues who have also fallen ill (Maunder et al. 2003; Greenbaum 2020). Decisions have to be made fast, ranging from efficiently triaging and isolating patients with suspicion of infection, to deciding whether to shut down departments and operating theatres when a patient or staff member tests positive, all this whilst being on limited resources. The pressure to act timely and to successfully diagnose, isolate and treat has been overwhelming, especially amid intense public and media scrutiny. This is in concordance with experience in other countries (Koinis et al. 2015).

Previous experience from SARS and H1N1 epidemics underlines that the psychological strain on healthcare professionals, who find themselves at the frontline of attempts to quell the outbreak, is significant (Huang et al. 2004; Goulia et al. 2010). Research into the psychological effects of infectious disease outbreaks such as SARS and pandemic flu (H1N1) shows consistent patterns of reactions and covers the experiences of staff in work, those in quarantine and those returning to work from time away sick. In the early rapid expansion phase of the SARS outbreak, similar to the current course of the COVID-19 pandemic, healthcare professionals reported feelings of extreme vulnerability, uncertainty and threat to life, alongside somatic and cognitive symptoms of anxiety (Huang et al. 2004), whilst during the $2009 \mathrm{H} 1 \mathrm{~N} 1$ pan- 
Table 8. Association between anxiety, socio-demographic and personal factors

\begin{tabular}{|c|c|c|c|c|}
\hline \multirow{2}{*}{$\begin{array}{l}\text { Socio-demographic and } \\
\text { personal factors }\end{array}$} & \multirow[t]{2}{*}{ Total $(N=302)$} & \multicolumn{2}{|c|}{ DASS Anxiety } & \multirow[t]{2}{*}{$\chi^{2}$ and $p$-value } \\
\hline & & Absent & Present & \\
\hline \multicolumn{4}{|l|}{ Age } & \multirow{6}{*}{$\begin{array}{l}\chi^{2}=4.84 \\
p=0.30\end{array}$} \\
\hline $21-30$ years & 176 & 135 & 41 & \\
\hline 31-40 years & 90 & 76 & 14 & \\
\hline 41-50 years & 11 & 9 & 2 & \\
\hline $51-60$ years & 15 & 12 & 3 & \\
\hline$>60$ years & 10 & 10 & 0 & \\
\hline \multicolumn{4}{|l|}{ Gender } & \multirow{3}{*}{$\begin{array}{l}\chi^{2}=0.0 \\
p=0.98\end{array}$} \\
\hline male & 189 & 152 & 37 & \\
\hline female & 113 & 90 & 23 & \\
\hline \multicolumn{4}{|l|}{ Religion } & \multirow{5}{*}{$\begin{array}{l}\chi^{2}=1.99 \\
p=0.57\end{array}$} \\
\hline Hindu & 281 & 223 & 58 & \\
\hline Muslim & 15 & 14 & 1 & \\
\hline Christian & 5 & 4 & 1 & \\
\hline others & 1 & 1 & 0 & \\
\hline \multicolumn{4}{|l|}{ Marital status } & \multirow{5}{*}{$\begin{array}{l}\chi^{2}=10.06 \\
p=0.018\end{array}$} \\
\hline single & 148 & 111 & 37 & \\
\hline married & 150 & 129 & 21 & \\
\hline divorced/separated & 3 & 2 & 1 & \\
\hline widow/widower & 1 & 0 & 1 & \\
\hline \multicolumn{4}{|l|}{ Family types } & \multirow{3}{*}{$\begin{array}{l}\chi^{2}=0.14 \\
p=0.71\end{array}$} \\
\hline nuclear & 165 & 134 & 31 & \\
\hline joint & 137 & 108 & 29 & \\
\hline \multicolumn{4}{|l|}{ History of physical illnesses } & \multirow{7}{*}{$\begin{array}{l}\chi^{2}=3.07 \\
p=0.69\end{array}$} \\
\hline none & 237 & 203 & 52 & \\
\hline cardiac illness & 13 & 9 & 4 & \\
\hline respiratory illnesses & 13 & 5 & 0 & \\
\hline diabetes & 5 & 11 & 2 & \\
\hline cardiac + diabetes & 1 & 13 & 2 & \\
\hline others & 15 & 1 & 0 & \\
\hline \multicolumn{4}{|c|}{ Past history of psychiatric illness } & \multirow{4}{*}{$\begin{array}{l}\chi^{2}=3.84 \\
p=0.14\end{array}$} \\
\hline yes & 13 & 11 & 2 & \\
\hline no & 277 & 224 & 53 & \\
\hline maybe & 12 & 7 & 5 & \\
\hline
\end{tabular}

DASS - Depression, Anxiety and Stress Scale

demic more than half of healthcare workers in a Greek tertiary hospital reported moderately high anxiety and subsequent psychological distress (Goulia et al. 2010).

It has been seen in a previous study that health professionals often have better awareness, positive attitudes towards epidemics/ pandemics and they often experience low levels of anxiety (Mishra et al. 2016). However, a study from Ethiopia reported poor knowledge and erroneous beliefs of healthcare professionals, during the Ebola virus outbreak in 2015, and it urged for intense training of healthcare professionals (Abebe et al. 2016). Health care workers at a hospital with intense liaison psychiatric services felt less psychological impact. Health care workers at a hospital that provided staff with less frequent information about the pandemic, felt unprotected. Workers in work environments that had a high risk of infection felt more anxious and more exhausted. In the fight against the 2019 novel coronavirus, medical workers have been facing enormous pressure, including a high risk of infection and 
Table 9. Association between work-related factors and anxiety

\begin{tabular}{|c|c|c|c|c|}
\hline \multirow[t]{2}{*}{ Work-related details } & \multirow{2}{*}{$\begin{array}{c}\text { Total } \\
(N=302)\end{array}$} & \multicolumn{2}{|c|}{ DASS Anxiety } & \multirow{2}{*}{$\begin{array}{l}\chi^{2} \text { and } \\
p \text {-value }\end{array}$} \\
\hline & & Absent & Present & \\
\hline \multicolumn{4}{|l|}{ Working post } & \multirow{6}{*}{$\begin{array}{l}\chi^{2}=10.53 \\
p=0.032\end{array}$} \\
\hline intern doctors & 57 & 43 & 14 & \\
\hline resident doctors & 87 & 66 & 21 & \\
\hline consultants & 114 & 101 & 13 & \\
\hline nursing staff & 9 & 5 & 4 & \\
\hline others & 35 & 27 & 8 & \\
\hline \multicolumn{4}{|l|}{ Work experience } & \multirow{5}{*}{$\begin{aligned} \chi^{2} & =3.60 \\
p & =0.31\end{aligned}$} \\
\hline$<1$ year & 82 & 61 & 21 & \\
\hline $1-3$ years & 57 & 44 & 13 & \\
\hline$>3-10$ years & 79 & 66 & 13 & \\
\hline$>10$ years & 84 & 71 & 13 & \\
\hline \multicolumn{4}{|l|}{ Duty type } & \multirow{3}{*}{$\begin{aligned} \chi^{2} & =0.002 \\
p & =0.96\end{aligned}$} \\
\hline fixed hours & 284 & 228 & 56 & \\
\hline shift duties & 18 & 14 & 4 & \\
\hline \multicolumn{4}{|l|}{ Duty hours/day } & \multirow{5}{*}{$\begin{array}{l}\chi^{2}=2.58 \\
p=0.76\end{array}$} \\
\hline$\leq 5$ hours & 31 & 28 & 3 & \\
\hline $6-8$ hours & 197 & 156 & 41 & \\
\hline$>8-12$ hours & 61 & 50 & 11 & \\
\hline$>12-24$ hours & 13 & 11 & 2 & \\
\hline \multicolumn{4}{|l|}{ Duty hours/week } & \multirow{3}{*}{$\begin{array}{l}\chi^{2}=1.90 \\
p=0.167\end{array}$} \\
\hline$\leq 48$ hours & 187 & 155 & 32 & \\
\hline$>48$ hours & 115 & 87 & 28 & \\
\hline \multicolumn{4}{|l|}{ Working area } & \multirow{7}{*}{$\begin{array}{l}\chi^{2}=16.65 \\
p=0.0052\end{array}$} \\
\hline COVID-19 duties & 73 & 47 & 26 & \\
\hline non-COVID-19 routine consultations & 130 & 110 & 20 & \\
\hline COVID-19 duties + non-COVID-19 routine consultations & 21 & 16 & 5 & \\
\hline emergency duties (ICU, CCU, PICU, NICU, etc.) & 62 & 55 & 7 & \\
\hline lab/paramedics/field duties & 9 & 8 & 1 & \\
\hline administration & 7 & 6 & 1 & \\
\hline
\end{tabular}

DASS - Depression, Anxiety and Stress Scale, ICU - Intensive Care Unit, CCU - Critical Care Unit, PICU - Pediatric Intensive Care Unit, NICU Neonatal Intensive Care Unit

Table 10. Fear of COVID-19 Scale

\begin{tabular}{lccccccc} 
& \multicolumn{7}{c}{ Questions } \\
\cline { 2 - 9 } Answers & $\mathbf{1}$ & $\mathbf{2}$ & $\mathbf{3}$ & $\mathbf{4}$ & $\mathbf{5}$ & $\mathbf{6}$ & $\mathbf{7}$ \\
Strongly disagree & 47 & 57 & 127 & 118 & 84 & 151 & 140 \\
\hline Disagree & 95 & 113 & 130 & 101 & 115 & 119 & 115 \\
\hline Can't say & 55 & 37 & 30 & 39 & 23 & 18 & 23 \\
\hline Agree & 94 & 88 & 11 & 37 & 71 & 12 & 19 \\
\hline Strongly agree & 11 & 7 & 4 & 7 & 9 & 2 & 5 \\
\hline
\end{tabular}

inadequate protection from contamination, symptoms, insomnia, denial, anger, and fear. overwork, frustration, discrimination, isolation, These mental health problems not only affect patients with negative emotions, and a lack the medical workers' attention, understandof contact with their families, and exhaustion. ing, and decision making ability, which might The severe situation is causing mental health hinder the fight against COVID-19, but could problems such as stress, anxiety, depressive also have a lasting effect on their overall well- 
being. Protecting the mental health of these medical workers is thus important for control of the epidemic and their own long-term health. On January 27, 2020, the National Health Commission of China published a national guideline of psychological crisis intervention for 2019 - nCoV (National Health Commission of the People's Republic of China, 2020). A few recent reports have shown that provision of adequate resources (e.g., medical supplies) and mental health support will bolster individual self-efficacy and confidence (Peter et al. 2020; Greenberg et al. 2020).

\section{Conclusions}

We observed significant psychological impacts of the COVID-19 pandemic on HCWs. A few risk factors such as female gender, single marital status, past history of psychiatric illness, working as interns and residents doctors with lesser experience and high risk duty areas such as COVID-19 duties in a particular situation were significantly associated with psychological disturbances such as stress, depression and anxiety. So supporting the mental health of HCWs is a critical part of the public health response to maintain an adequate workforce and to maximize the ability of HCWs to face this pandemic. Also they need to be assessed periodically for the physical and psychological impact of this crisis and need to receive help in those areas which are disturbed.

\section{Acknowledgements}

This is a cross sectional study with convenience sampling, so the results cannot be generalized. Forms were distributed via email, WhatsApp messages and Messenger, so the exact response rate cannot be determined. As self-reported scales were used, subjective bias may exist.

\section{References}

1. Abebe TB, Bhagavathula AS, Tefera YG, et al. Healthcare professionals' awareness, knowledge, attitudes, perceptions and beliefs about ebola at Gondar University Hospital, Northwest Ethiopia: a cross-sectional study. J Public Health Afr 2016; 7: 570.

2. Adshead G. Healing ourselves: ethical issues in the care of sick doctors. Adv Psychiatr Treat 2005; 11: 330-337.

3. Ahorsu DK, Lin CY, Imani V, et al. The fear of COVID-19 scale: development and initial validation. Int J Ment Health Addict 2020; 1-9. doi: 10.1007/s11469-020-00270-8.

4. Andersen KG, Rambaut A, Lipkin WI, et al. The proximal origin of SARS-CoV-2. Nat Med 2020; 26: 450-452.

5. Aoyagi Y, Beck CR, Dingwall R, et al. Healthcare workers' willingness to work during an influenza pandemic: a sys- tematic review and meta-analysis. Influenza Other Respir Viruses 2015; 9: 120-130.

6. Brooks SK, Dunn R, Amlôt R, et al. A systematic, thematic review of social and occupational factors associated with psychological outcomes in healthcare employees during an infectious disease outbreak. J Occup Environ Med 2018; 60: 248-257.

7. Brooks SK, Webster RK, Smith LE, et al. The psychological impact of quarantine and how to reduce it: rapid review of the evidence. Lancet 2020; 395: 912-920.

8. Chambers R, Belcher J. Self-reported health care over the past 10 years: a survey of general practitioners. $\mathrm{Br} J \mathrm{Gen}$ Pract 1992; 42: 153-156.

9. Chatterjee S, Bhattacharyya R, Bhattacharyya $S$, et al. Attitude, practice, behaviour and impact of COVID-19 on doctors. Indian J Psychiatr 2020; 62: 257-265.

10. Chong MY, Wang WC, Hsieh WC, et al. Psychological impact of severe acute respiratory syndrome on health workers in a tertiary hospital. Br J Psychiatry 2004; 185: 127-133.

11. Cohen S, Kamarck T, Mermelstein R. A global measure of perceived stress. J Health Soc Behav 1983; 24: 386-396.

12. Deblina R, Sarvodaya T, Sujita Kumar K, et al. Study of knowledge, attitude, anxiety \& perceived mental healthcare need in Indian population during COVID-19 pandemic. Asian J Psychiatr 2020; 51: 1876-2018.

13. Forsythe M, Calnan M, Wall B. Doctors as patients: postal survey examining consultants and general practitioners adherence to guidelines. BMJ 1999; 319: 605-608.

14. Goulia P, Mantas C, Dimitroula D, et al. General hospital staff worries, perceived sufficiency of information and associated psychological distress during the A/H1N1 influenza pandemic. BMC Infect Dis 2010; 10: 322.

15. Greenbaum Z. Psychologist leads innovative approach to tackle psychological toll of COVID-19. Am Psychol Assoc 2020.

16. Greenberg N, Docherty M, Gnanapragasam S, et al. Managing mental health challenges faced by healthcare workers during covid-19 pandemics. BMJ 2020; 368: m1211.

17. Huang OL, Wen JK, Chen CL. Psychological impact of severe acute respiratory syndrome on health workers in a tertiary hospital. Br J Psychiatry 2004; 185: 127-133.

18. Huang JZ, Han MF, Luo TD, et al. Mental health survey of 230 medical staff in a tertiary infectious disease hospital for COVID-19. Zhonghua Lao Dong Wei Sheng Zhi Ye Bing Za Zhi 2020; 38: E001.

19. Koinis A, Giannou V, Drantaki V, et al. The impact of healthcare workers job environment on their mental-emotional health. coping strategies: the case of a local general hospital. Health Psychol Res 2015; 3: 1984.

20. Kushal A, Gupta S, Mehta M, et al. Study of stress among health care professionals: a systemic review. Int J Res Found Hospital Health Care Adm 2018; 6: 6-11.

21. Lai J, Ma S, Wang Y, et al. Factors associated with mental health outcomes among health care workers exposed to coronavirus disease 2019. JAMA 2020; 3: e203976.

22. Lovibond SH, Lovibond PF. Manual for the depression anxiety stress scales. $2^{\text {ed }}$ ed. Psychology Foundation of Australia, Sydney 1995.

23. Maunder R, Hunter J, Vincent L, et al. The immediate psychological and occupational impact of the 2003 SARS outbreak in a teaching hospital. CMAJ 2003; 168: 1245-1251.

24. Mishra P, Bhadauria US, Dasar PL, et al. Knowledge, attitude and anxiety towards pandemic flu a potential bio weapon among health professionals in Indore City. Przegl Epidemiol 2016; 70: 125-127. 
25. National Health Commission of the People's Republic of China. The guideline of psychological crisis intervention for 2019- nCoV pneumonia [Internet] (cited Jan 27, 2020). Available from: http://www.nhc.gov.cn/jkj/s3577/ 202001/6adc 08b966594253b2b791be5c3b9467.shtml.

26. Peter EW, Rima S, Wayne L. Mitigating the psychological effects of COVID-19 on health care workers. CMAJ 2020, 192: E459-E460.

27. Tan BYQ, Chew NWS, Lee GKH, et al. Psychological impact of the COVID-19 pandemic on health care workers in Singapore. Ann Intern Med 2020; 173: 317-320.

28. Velavan TP, Meyer CG. The COVID-19 epidemic. Trop Med Int Health 2020; 25: 278-280.

29. World Health Organization. WHO announces COVID-19 outbreak a pandemic [Internet] (cited April 3, 2020). Available from: http://www.euro.who.int/en/health-topics/health-emergencies/coronavirus-covid-19/news/ news/2020/3/who-announces-covid-19-outbreak-a-pandemic.

30. Xiao H, Zhang Y, Kong D, et al. The effects of social support on sleep quality of medical staff treating patients with coronavirus disease 2019 (COVID-19) in January and February 2020 in China. Med Sci Monit 2020; 26: e923549.

31. Zhang W, Wang K, Yin L, et al. Mental health and psychosocial problems of medical health workers during the COVID-19 epidemic in China. Psychother Psychosom 2020; 89: 242-250. 